\title{
PD-1 inhibition in malignant melanoma and lack of clinical response in chronic lymphocytic leukemia in the same patients: a case series
}

\author{
I. Landego MD, ${ }^{*}$ D. Hewitt RN, ${ }^{\dagger}$ I. Hibbert BN, ${ }^{\ddagger}$ D. Dhaliwal $M D, \$$ W. Pieterse $M D, \|$ \\ D. Grenier $\mathrm{BS}_{\mathrm{c}} \mathrm{MD}, \stackrel{\S}{\S}$. Wong $\mathrm{BSc}_{\mathrm{c}} \mathrm{MD},{ }^{\S}$ J. Johnston $\mathrm{MB} \mathrm{BCh}^{+\S}$ and V. Banerji $\mathrm{MD}^{\dagger \S}$
}

\begin{abstract}
Chronic lymphocytic leukemia (CLL) is the most common adult leukemia in the Western world. Unfortunately, affected patients are often immunosuppressed and at increased risk of infection and secondary malignancy. Previous meta-analysis has found that patients with CLL have a risk of melanoma that is increased by a factor of 4 compared with the general population. Recent advances in the understanding of the PD receptor pathway have led to immunotherapies that target cancer cells. The use of PD-1 inhibitors is now considered first-line treatment for BRAF wild-type metastatic melanoma. Interestingly, early preclinical data suggest that inhibition of that pathway could also be used in the treatment of CLL; however, recent clinical data did not support the effectiveness of that approach. In this case series, we highlight 2 cases in which patients with CLL and concurrent malignant melanoma underwent treatment with PD-1 inhibitors and were found to experience reductions in their white blood cell counts without improvement in their hemoglobin. Those cases further illustrate that treatment of CLL with PD-1 inhibitors is ineffective.
\end{abstract}

Key Words Immunotherapy, chronic lymphocytic leukemia, CLL, melanoma

Curr Oncol. 2020 June:27(3)169-172

www.current-oncology.com

\section{INTRODUCTION}

Chronic lymphocytic leukemia (CLL) is the most common adult leukemia. It is considered to be a heterogeneous disease, with some patients undergoing treatment at the time of diagnosis, and others having a more indolent asymptomatic clinical course. One of the major complications of CLL is an increased risk of secondary malignancies ${ }^{1-3}$. That propensity is in part attributable to the immunodeficiency imparted by CLL itself and the toxicity of the chemotherapy regimens used ${ }^{4}$. Melanoma is considered to be an immunogenic cancer, which puts individuals who are immunocompromised at increased risk ${ }^{5,6}$. Interestingly, a systematic review and meta-analysis performed by Olsen et al. ${ }^{7}$ found that melanoma was increased by a factor of 4 in patients with CLL compared with the general population. Although there is an increased risk of co-malignancy with CLL and melanoma, the treatment strategies are different.
Within the past decade, the treatment of metastatic melanoma has been revolutionized by the advent of effective molecular and immunomodulatory therapies ${ }^{8}$. The treatment regimens for metastatic melanoma are determined by the presence or absence of a driver mutation. If a driver mutation is present, directed molecular therapies targeting BRAF are considered for first-line therapy. In patients who are $B R A F$ wild-type, immunomodulation can be used to harness the immune response against cancer cells ${ }^{8}$. Specifically, the PD-1 inhibitor nivolumab has been shown to be more effective, being associated with increased progression-free and overall survival and with fewer adverse effects than are seen with the traditional chemotherapy, dacarbazine ${ }^{9}$. Additionally, increased overall survival has been associated with the PD-1 inhibitor pembrolizumab compared with the CTLA- 4 inhibitor ipilimumab ${ }^{10}$. Interestingly, inhibition of PD-1 or PD-L1 has shown some preclinical effectiveness in CLL ${ }^{11}$. 
The PD-1 protein (CD279) is a costimulatory molecule that functions as an immune checkpoint to help downregulate aberrant responses ${ }^{12}$. It is expressed on all cells in the lymphoid lineage, including B, T, and natural killer cells ${ }^{12}$. It is well established that patients with CLL have profound $\mathrm{T}$ cell defects ${ }^{13,14}$. When compared with control donors, patients with CLL had significantly higher levels of PD-1 on CD4 and CD8 T lymphocytes ${ }^{15}$. Also, compared with CD19+ lymphocytes from control donors, CD19+ lymphocytes from patients with CLL expressed higher levels of PD-L1 ${ }^{15}$. It has therefore been proposed that manipulation of that signalling axis might help to restore immune function in patients with CLL.

In the present case series, we describe 2 patients with concurrent metastatic melanoma and CLL who were treated with PD-1 inhibitors for their melanoma. Interestingly, we found that both patients experienced initial reductions in their white blood cell (WBC) counts, but unfortunately, their CLL did not respond.

\section{CASE DESCRIPTION}

Using the CancerCare Manitoba electronic record, the cases presented here were retrospectively reviewed. Consent for the case reports was obtained from the patients and their families.

\section{Case 1}

An 82-year-old woman with a history of a right calf lesion resected in 2005 and determined to be an indeterminate nevus was subsequently diagnosed in 2006 with CLL, which was monitored with a watch-and-wait approach. In 2011, the patient developed a $7.5 \mathrm{~mm}$ malignant melanoma on her right posterior medial calf, for which she received wide local excision without sentinel node biopsy.

Because of increasing lymphocytosis, the patient was started on chlorambucil for her CLL in 2011. Despite dose escalation, the CLL progressed to Rai stage IV by March 2016. Unfortunately, around the same time she also developed several blue discolored lesions around the previous surgical site (Figure 1), which were determined to be recurrent blue nevi metastatic melanoma, $B R A F$ wild-type.

There was concern that treatment of the CLL would cause immunosuppression and progression of the melanoma. As a result, immunomodulation with the PD-1 inhibitor nivolumab, which has been shown to be effective in treating melanoma, was started. The patient then received 3 cycles of nivolumab, which she tolerated quite well.

Initially, it was thought that the CLL was responding, because the patient's WBC counts declined to $159 \times 10^{9} / \mathrm{L}$ from $240 \times 10^{9} / \mathrm{L}$ (Figure 2 ). However, she became symptomatic from her anemia. The decision was made to stop nivolumab and to commence CLL-directed treatment with obinutuzumab and chlorambucil. For the melanoma, the patient was switched to localized interleukin 2 injections to the lesions near the previous excision site.

\section{Case 2}

An 87-year-old woman with a history of lentigo maligna on the left naris that had previously been excised with skin grafting, was subsequently diagnosed with Rai stage 0 CLL

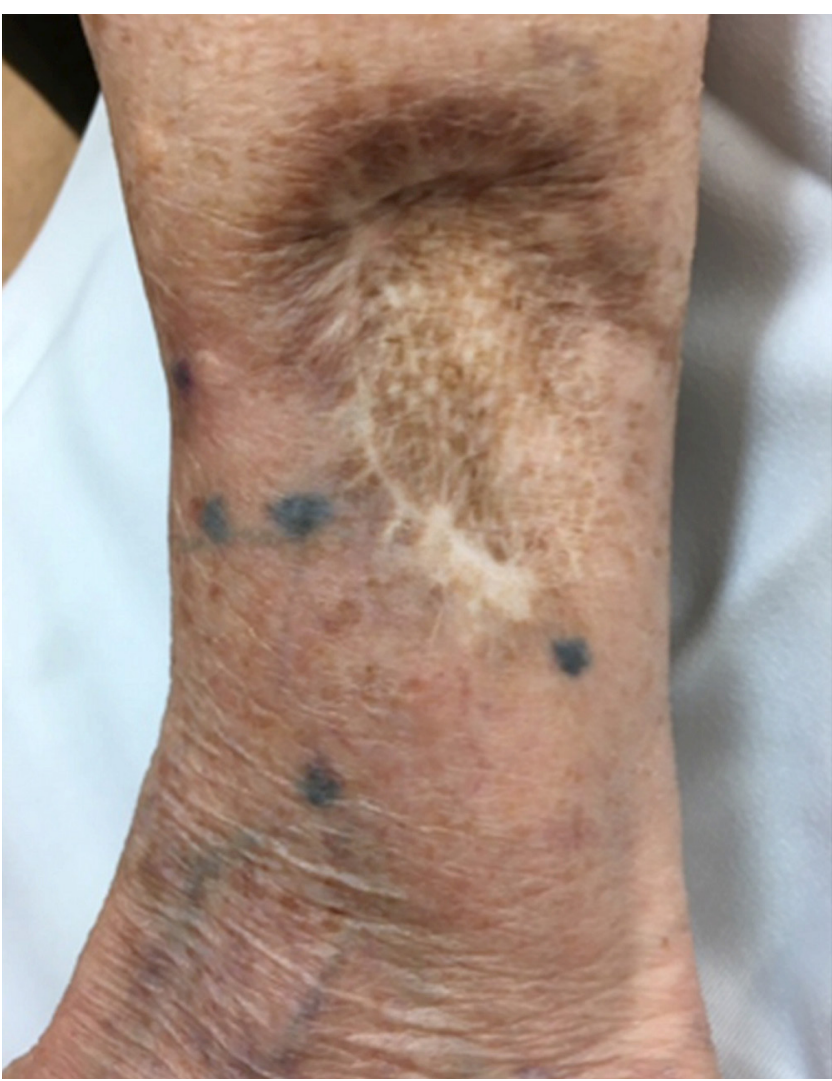

FIGURE 1 Recurrent melanoma, right calf, in case 1.

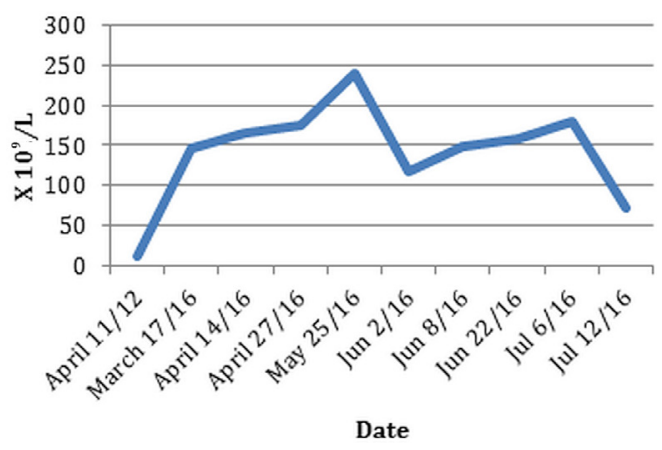

FIGURE 2 White blood cell (WBC) counts, case 1. Dates of treatment with nivolumab were 26 May, 9 June, and 23 June 2016.

in September 2009. At that time, she was asymptomatic, with monitoring by regular blood counts.

The patient remained on active observation until July 2014, when she developed Rai stage III CLL with lymphadenopathy, splenomegaly, and anemia. At the same time, she had a melanoma recurrence involving the entire left nasal wall (Figure 3). The patient was initially started on imiquimod, but definitive therapy with excision was ultimately required.

Pathology revealed a $B R A F$ wild-type malignant melanoma with a Breslow depth of $8.5 \mathrm{~mm}$ and margins positive for in situ and invasive disease. The patient then started on localized treatment with interleukin 2 injections. 


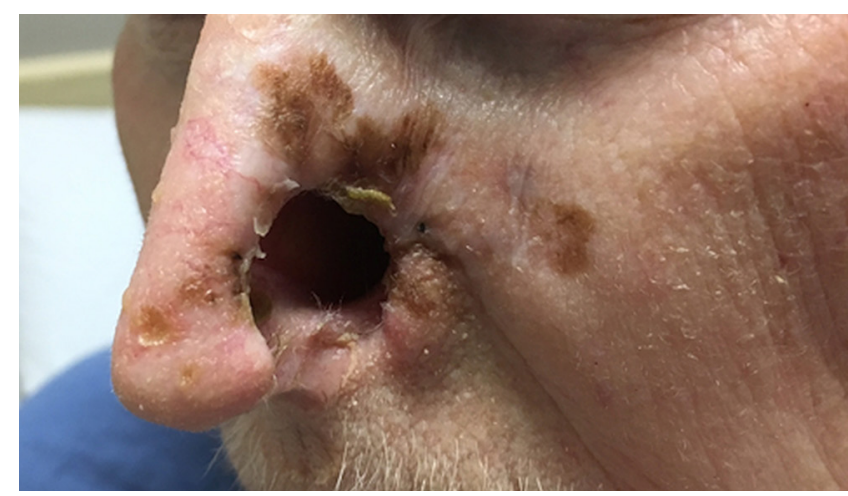

FIGURE 3 Patient after resection of melanoma, case 2.

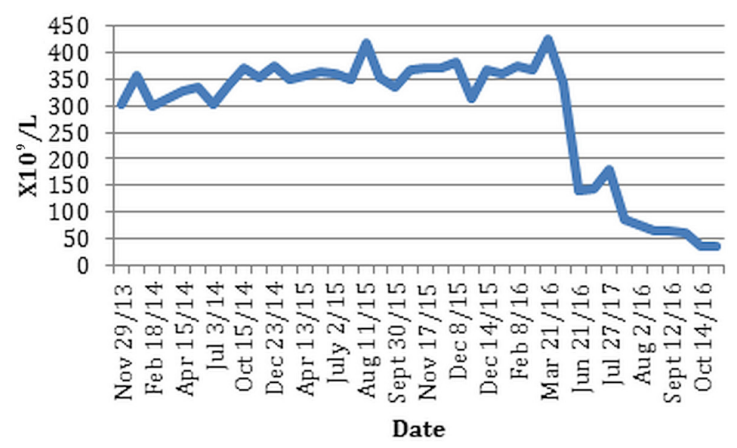

FIGURE 4 White blood cell (WBC) counts, case 2. Dates of treatment with pembrolizumab were 28 June, 18 July, 9 August, 30 August, and 20 September 2016.

Unfortunately, she developed pleural effusions that were positive for melanoma cells, indicating stage IV disease.

Given this patient's immunocompromised state and progression of her melanoma, the patient was started on pembrolizumab on 6 June 2016. Interestingly, by 9 June, her WBC count had declined to $167 \times 10^{9} / \mathrm{L}$ from $235 \times 10^{9} / \mathrm{L}$. She continued treatment with pembrolizumab every 3 weeks, and by the end of cycle 4 , her WBC count had come down to $62 \times 10^{9} / \mathrm{L}$ (Figure 4 ).

The patient remained symptomatic and transfusiondependent because of her anemia, and passed away in hospital.

\section{DISCUSSION}

Inhibition of the PD-1/PD-L1 signalling axis has been found to be effective in preclinical models of CLL. In this case series, we describe 2 patients with concurrent malignant melanoma and CLL who underwent treatment with PD-1 inhibitors and who were found to experience reductions in their lymphocyte (WBC) counts, with progressive anemia. To our knowledge, those observations provide some of the first clinical evidence (outside of trials) that PD-1 inhibitors are ineffective in CLL.

Tumour cells have evolved several mechanisms to evade the host's immune system by disruption of immune checkpoints. The PD-1/PD-L1 pathway, which functions to maintain tolerance and prevent autoimmune reactions, is often disrupted in various malignancies. Preclinical studies found that transgenic expression of PD-L1 by tumour cells rendered them less susceptible to T cell-mediated lysis and increased their tumorigenicity ${ }^{16}$. The binding of PD-1 to PD-L1 has been shown to hamper T cell-mediated responses, including proliferation, cytokine production, and cytotoxic effects ${ }^{11}$. Taken together, those results suggest that targeting the $\mathrm{PD}-1 / \mathrm{PD}-\mathrm{Ll}$ pathway might be an effective therapeutic approach for targeting cancer cells.

It is well established that patients with CLL have profound immune deficits. That observation might in part be attributable to overexpression of PD-L1 by CLL cells ${ }^{17}$. Preclinical data from McClanahan et al. ${ }^{11}$, who used the E $\mu$-TCLl CLL mouse model, found that blocking PD-Ll resulted in improved $\mathrm{T}$ cell function and restoration of myeloid cells. Those results highlight the importance and therapeutic potential of the PD-1/PD-L1 pathway in CLL treatment.

In a recent study by Ding et al ${ }^{18}$, patients with relapsed or refractory CLL treated with pembrolizumab did not experience a clinical response, but patients with Richter transformation did selectively experience clinical responses. Similarly, patients with refractory or relapsed Hodgkin lymphoma treated with the PD-1 antibody nivolumab showed significant clinical response rates ${ }^{19}$.

When taken together with foregoing studies, our case series provides the first clinical evidence to suggest that PD-1 inhibitors might be effective in lowering the $\mathrm{WBC}$ count in patients with CLL, but might not be effective as single-agent therapy.

Although there might be genetic associations linking melanoma and CLL, it is likely that the immunosuppressed state of either condition predisposes patients to concurrent malignancy ${ }^{7}$. One major limitation of our case series is that each patient failed to complete a course of treatment because of CLL disease progression. Although both patients experienced reductions in their WBC counts, it is unclear whether PD-1 inhibition had any effect on the lymphadenopathy or organomegaly in either patient.

\section{SUMMARY}

This case series highlights 2 patients with concurrent malignant melanoma and CLL who were treated with PD-1 inhibitors. Although PD-1 inhibitors are the first-line therapy for malignant melanoma, they might not be independently effective for CLL. Interestingly, CLL samples with the Richter transformation have the highest levels of PD-1 expression ${ }^{20}$. In both cases, a clinical response with reduction in the patient's WBC count occurred, but unfortunately, either because of progression of the underlying solid tumour or ineffective control of bone marrow, treatment did not yield the expected benefits. Currently, evaluations of combination strategies with novel targeted agents such as ibrutinib or venetoclax in combination with immunotherapy are ongoing (see NCT02846623 and NCT03514017 at https://ClinicalTrials.gov/). Additionally, a trial for patients with the Richter transformation, using treatment with immunotherapy in combination with venetoclax and obinutuzumab is also recruiting (NCT02846623). And safety data in a phase I-II study of ibrutinib-nivolumab in relapsed refractory CLL has been published ${ }^{21}$. 
Our case series provides clinical evidence that, as a single agent, PD-1 blockade is ineffective in CLL, supporting further investigation into the use of PD-1/PD-L1 inhibitors in combination for the treatment of CLL.

\section{CONFLICT OF INTEREST DISCLOSURES}

We have read and understood Current Oncology's policy on disclosing conflicts of interest, and we declare that we have none.

\section{AUTHOR AFFILIATIONS}

*Department of Internal Medicine, Rady College of Medicine, Max Rady Faculty of Health Sciences, University of Manitoba, ${ }^{\dagger}$ Research Institute of Oncology and Hematology, CancerCare Manitoba and the University of Manitoba, ${ }^{\ddagger}$ Department of Nursing, CancerCare Manitoba, ${ }^{\S}$ Section of Hematology and Oncology, Rady College of Medicine, Max Rady Faculty of Health Sciences, University of Manitoba, and "Russell Health Centre, Community Oncology Program, Winnipeg, MB.

\section{REFERENCES}

1. Rozman C, Montserrat E. Chronic lymphocytic leukemia. NEngl J Med 1995;333:1052-7.

2. Kipps TJ, Stevenson FK, Wu CJ, et al. Chronic lymphocytic leukaemia. Nat Rev Dis Primers 2017;3:16096.

3. Beiggi S, Banerji V, Deneka A, Griffith EJ, Gibson SB, Johnston JB. Comparison of outcome between referred and non-referred chronic lymphocytic leukemia patients to a specialized CLL clinic: a Canadian-based study. Cancer Med 2016;5:1-9.

4. Hallek M, Fischer K, Fingerle-Rowson G, et al. on behalf of the International Group of Investigators and the German Chronic Lymphocytic Leukaemia Study Group. Addition of rituximab to fludarabine and cyclophosphamide in patients with chronic lymphocytic leukaemia: a randomised, openlabel, phase 3 trial. Lancet 2010;376:1164-74.

5. Davis ID. Immunotherapy of melanoma. Cancer Forum 2002; 26:115-17.

6. Olsen CM, Knight LL, Green AC. Risk of melanoma in people with HIV/AIDS in the pre- and post-HAART eras: a systematic review and meta-analysis of cohort studies. PLoS One 2014;9:e95096.

7. Olsen CM, Lane SW, Green AC. Increased risk of melanoma in patients with chronic lymphocytic leukaemia: systematic review and meta-analysis of cohort studies. Melanoma Res 2016;26:188-94.

8. Lenehan JG, Ernst DS. Progress in the treatment of metastatic cutaneous melanoma. Oncology Exchange 2015;14:20-8.

9. Ascierto PA, Long GV, Robert C, et al. Survival outcomes in patients with previously untreated $B R A F$ wild type advanced melanoma treated with nivolumab therapy. Three year follow-up of a randomized phase 3 trial. JAMA Oncol 2019;5:187-94.

10. Schacter J, Ribas A, Long GV, et al. Pembrolizumab versus ipilimumab for advanced melanoma: final overall survival results of a multicentre, randomised, open-label phase 3 study (KEYNOTE-006). Lancet 2017;390:1853-62.

11. McClanahan F, Hanna B, Miller S, et al. PD-L1 checkpoint blockade prevents immune dysfunction and leukemia development in a mouse model of chronic lymphocytic leukemia. Blood 2015;126:203-11.

12. Topalian SL, Taube JM, Anders RA, Pardoll DM. Mechanismdriven biomarkers to guide immune checkpoint blockade in cancer therapy. Nat Rev Cancer 2016;16:275-87.

13. Ramsay AG, Johnson AJ, Lee AM, et al. Chronic lymphocytic leukemia $\mathrm{T}$ cells show impaired immunological synapse formation that can be reversed with an immunomodulating drug. J Clin Invest 2008;118:2427-37.

14. Riches JC, Davies JK, McClanahan F, et al. T cells from CLL patients exhibit features of T-cell exhaustion but retain capacity for cytokine production. Blood 2017;121:1612-21.

15. Brusa D, Serra S, Coscia M, et al. The PD-1/PD-L1 axis contributes to T-cell dysfunction in chronic lymphocytic leukemia. Haematologica 2013;98:953-63.

16. Iwai $\mathrm{Y}$, Ishida $\mathrm{M}$, Tanaka $\mathrm{Y}$, Okazaki T, Honjo T, Minato N. Involvement of PD-L1 on tumor cells in the escape from host immune system and tumor immunotherapy by PD-L1 blockade. Proc Natl Acad Sci U S A 2002;99:12293-7.

17. Ramsay AG, Clear AJ, Fatah R, Gribben JG. Multiple inhibitory ligands induce impaired T-cell immunologic synapse function in chronic lymphocytic leukemia that can be blocked with lenalidomide: establishing a reversible immune evasion mechanism in human cancer. Blood 2012;120:1412-21.

18. Ding W, Laplant BR, Call TG, et al. Pembrolizumab in patients with CLL and Richter transformation or with relapsed CLL. Blood 2017;129:3419-27.

19. Ansell SM, Lesokhin AM, Borrello I, et al. PD-1 blockade with nivolumab in relapsed or refractory Hodgkin's lymphoma. N Engl J Med 2015;372:311-19.

20. He M, Ding W, Viswanatha DS, et al. PD-1 expression in chronic lymphocytic leukemia/small lymphocytic lymphoma (CLL/SLL) and large B-cell Richter transformation (DLBCL-RT): a characteristic feature of DLBCL-RT and potential surrogate marker for clonal relatedness. Am J Surg Pathol 2018;42:843-54.

21. Younes A, Brody J, Carpio C, et al. Safety and activity of ibrutinib in combination with nivolumab in patients with relapsed non-Hodgkin lymphoma or chronic lymphocytic leukaemia: a phase 1/2a study. Lancet Haematol 2019;6:e67-78. 